\title{
NOUVELle
}

\section{Un gène codant une métalloprotéase impliqué dans I'hétérotaxie}

Patrice Bouvagnet ${ }^{1}$, Anne Guimier ${ }^{2}$, Jeanne Amiel ${ }^{2}$, Christopher T. Gordon²

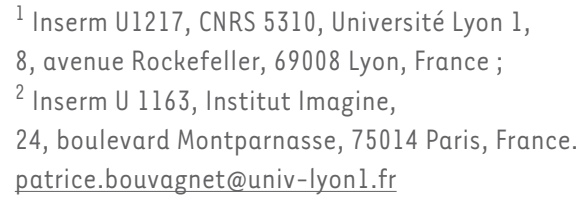

\section{L'hétérotaxie}

La latéralisation des organes thoraciques et abdominaux est un processus fondamental du développement embryonnaire. Elle survient après la mise en place des axes crânio-caudal et dorso-ventral. Le premier organe à se latéraliser est le cœur. Celui-ci n'est initialement qu'un tube, orienté selon l'axe crânio-caudal, dont la croissance longitudinale va excéder celle de l'embryon. II commence alors à former une courbe vers la droite, ce qui constitue le premier événement de latéralisation de l'embryon. Les arcs vasculaires qui sont situés entre le futur cœur et la région céphalique vont, eux aussi, avoir une évolution différente dans la partie droite et la partie gauche de l'embryon [1] $(\rightarrow)$.

L'intestin, lui, va subir un mouvement de rotation comme en témoigne son $(\rightarrow)$ Voir la Nouvelle de F. Bajolle et S. Zaffran, $m / s n^{\circ} 4$, avril 2008, page 354 parcours dans l'abdomen, et l'estomac va se retrouver du côté gauche et le foie du côté droit. Cette organisation finale est observée chez la majorité des individus. Cependant, pour certains, la latéralisation est parfaitement inversée : le cœur et l'estomac sont situés à droite, le foie à gauche. Cette inversion complète, en miroir, des organes thoraciques et abdominaux, appelée situs inversus totalis, est-elle pénalisante? La réponse est non. II n'y a aucun handicap à être parfaitement inversé, sauf qu'en cas d'appendicite, la douleur abdominale serait située à gauche et non à droite ! Cependant une inversion partielle de la latéralisation (ou situs ambiguus) est

une cause de morbidité et de mortalité, car presque toujours associée à une malformation cardiovasculaire [2] $(\rightarrow)$. Celle-ci peut être grave et nécessiter, pour assurer une survie, une chirurgie palliative afin d'ob-

$\rightarrow$ Voir la Synthèse de N. Diguet et S. Meilhac, $\mathrm{m} / \mathrm{s}$ $n^{\circ} 11$, novembre 2014, page 996

tenir une amélioration partielle de la circulation sanguine. L'inversion complète (situs inversus totalis) et l'inversion partielle (situs ambiguus) sont regroupées sous le terme d'hétérotaxie (heteros: différent et taxos : position), bien que les anglo-saxons préfèrent utiliser le terme hétérotaxie (heterotaxy) comme synonyme de situs ambiguus.

L'autre circonstance dans laquelle une hétérotaxie est pénalisante est lorsqu'elle est associée à des infections respiratoires récidivantes. Celles-ci sont dues à une dysfonction des cils situés au pôle apical des cellules épithéliales qui tapissent les voies aériennes supérieures (sinus) et inférieures (trachée, bronches). Le rôle de ces cils est d'évacuer, grâce à un mouvement équivalent à une hola dans un stade de football, les particules microbiennes que nous inhalons en respirant. Cette dysfonction est appelée dyskinésie ciliaire primitive. Quel est le lien entre l'hétérotaxie et la dyskinésie ciliaire? Les embryons de vertébrés ont presque tous une dépression à la surface de l'endoderme, appelée le nœud embryonnaire (et la vésicule de Küpffer chez les poissons), au fond duquel se trouve un tapis de cellules présentant un seul cil orienté vers la partie caudale de l'embryon (Figure IA).
Contrairement aux cils des muqueuses respiratoires qui ont un mouvement de type « essuie-glace », le monocil embryonnaire présente un mouvement de rotation qui génère un flux de liquide péri-embryonnaire de la droite vers la gauche de l'embryon (Figure 1B). Ce flux liquidien est responsable du début d'une signalisation moléculaire différentielle entre la droite et la gauche, bien avant l'apparition de la boucle cardiaque. Le dysfonctionnement de ce cil embryonnaire peut donc entraîner une hétérotaxie. Le lien entre l'hétérotaxie et la dyskinésie ciliaire primitive respiratoire est simplement le reflet de l'existence de protéines communes aux cils respiratoires et aux cils embryonnaires. En conséquence, une mutation qui altère la synthèse d'une protéine commune aux deux cils peut se traduire par une hétérotaxie et une dyskinésie ciliaire (syndrome de Kartagener [3]) $(\rightarrow)$.

La plupart des gènes qui ont, jusqu'à présent, $(\rightarrow)$ Voir la Synthèse de R. BachmannGagescu, $m / s n^{\circ} 11$, novembre 2014, page 1011 été impliqués dans l'hétérotaxie chez l'homme sont soit des gènes codant des protéines du cil embryonnaire, soit des gènes codant des protéines impliquées dans des étapes de la latéralisation en aval de l'étape du cil embryonnaire (pour une revue voir [4]).

\section{Le gène MMP21}

Dans une étude récente [5], des jumeaux dizygotes (un garçon et une fille), issus de parents non apparentés, présentaient tous les deux une malformation 


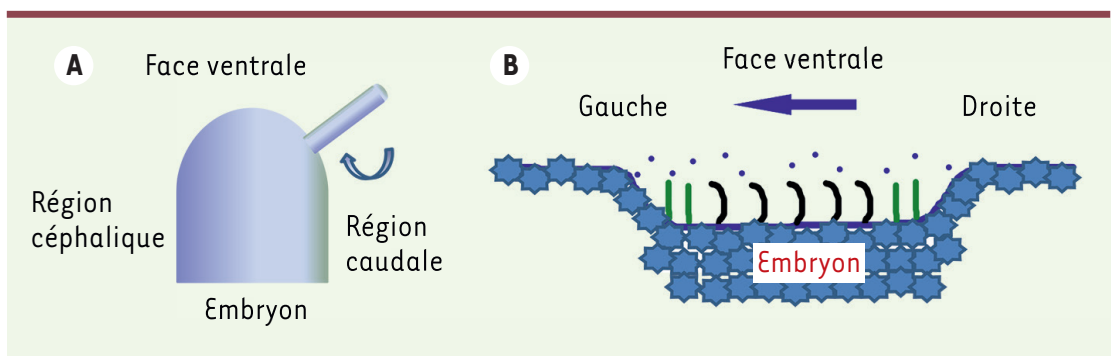

Figure 1. Schéma présentant le nœud embryonnaire et les cellules monociliées. A. Schéma d'une cellule tapissant le plancher du nœud embryonnaire. Son unique cil n'est pas implanté au sommet de la cellule et possède une orientation vers la région caudale. $\boldsymbol{B}$. Le mouvement de rotation des cils du nœud embryonnaire génère un courant de liquide péri-embryonnaire de la droite de l'embryon vers la gauche de l'embryon.

cardiovasculaire complexe, associée à une hétérotaxie et sans signe respiratoire laissant suggérer une atteinte des cils respiratoires. Un séquençage exomique (c'est-à-dire un séquençage de toutes les parties codant les protéines du génome humain) de l'ADN germinal des deux enfants a permis de découvrir une mutation d'origine paternelle et une mutation d'origine maternelle dans le gène MMP21. Après séquençage de ce gène dans une série de cas de malformations cardiovasculaires avec hétérotaxie (pour 154 patients) ou sans hétérotaxie (pour 110 patients), sept autres cas familiaux ont été identifiés, le plus souvent dans un contexte de consanguinité parentale ( $5 / 7$ cas). Pour deux de ces familles, un génotypage a été effectué permettant de confirmer que les malades étaient bien homozygotes dans la région génomique contenant le gène MMP21. Dans une des familles consanguines, trois membres de la famille étaient porteurs homozygotes de la mutation MMP21 familiale. L'un avait un situs inversus totalis et ne souffrait donc de rien, un autre avait une malformation cardiovasculaire pour laquelle il était traité et enfin, le dernier n'avait pas d'hétérotaxie (disposition classique des organes ou situs solitus) ni de malformation cardiovasculaire, démontrant que l'impact de ces mutations est extrêmement variable. Pour ces huit familles identifiées, aucun des malades ne souffraient d'autres signes que ceux liés à l'hétérotaxie, suggérant que les mutations du gène MMP21 n'altéraient pas d'autres mécanismes cellulaires que celui lié à la latéralisation. En plus de ces huit familles, un cas familial supplémentaire hispano-américain est rapporté dans l'article de Guimier et al. [5], ainsi que trois autres cas, dont deux familiaux, dans d'autres études indépendantes $[6,7]$.

MMP21 code une protéine probablement sécrétée car elle présente une région amino-terminale contenant un peptide signal ${ }^{1}$. Cette protéine a une structure similaire à d'autres métalloprotéases avec (1) un prodomaine auto-inhibiteur, qui est probablement clivé près du site de son activité, (2) un domaine catalytique peptidase et (3) quatre domaines hémopexine ${ }^{2}$ du côté carboxy-terminal, impliqués dans la reconnaissance du substrat de cette enzyme (Figure 2). L'expression de cette métalloprotéase est observée dans de multiples tissus embryonnaires et adultes, ainsi que dans des tumeurs cancéreuses [8]. Les mutations rapportées ont été trouvées dans tous les domaines de la protéine (Figure 2) [5-7], démontrant que celleci doit être intègre pour fonctionner normalement.

\footnotetext{
Chaîne peptidique d'une protéine servant à adresser celle-ci à un compartiment cellulaire particulier, comme les vésicules de sécrétion par exemple.

${ }^{2}$ Glycoprotéine se liant aux hèmes.
}

Différents modèles animaux ont permis d'étudier la fonction de MMP21. Tout d'abord, chez des embryons de poisson zèbre, I'utilisation de la technique d'hybridation in situ $^{3}$ a révélé que la transcription de MMP21 est strictement restreinte à la région de la vésicule de Küpffer (c'est-à-dire l'équivalent du nœud embryonnaire de l'homme et de la souris). De plus, l'injection de morpholinos $^{4}$ bloquant l'épissage ou la traduction de l'ARNm de MMP21, induit l'apparition d'anomalies de la boucle cardiaque chez les embryons de poisson zèbre au stade $48 \mathrm{~h}$ post-fertilisation. Chez la souris, des mutations du gène MMP21, obtenues soit par mutagénèse aléatoire chimique avec le $\mathrm{N}$-nitroso-N-éthylurée (ENU) [9], soit par mutagenèse ciblée avec la technologie CRISPR/ Cas9 [10] $(\rightarrow)$ provoquent une hétérotaxie. Son hérédité $\rightarrow$ Voir la Synthèse de J.P. Tremblay, $m / s n^{\circ} 11$, novembre 2015, page 1014 est récessive, comme chez l'homme, et une pénétrance incomplète ${ }^{5}$ a été observée dans le modèle CRISPR/Cas9. Dans ces modèles, le cil du nœud embryonnaire fonctionnait normalement [6].

Une étude phylogénique a montré que le gène MMP21 n'est pas fonctionnel dans le génome de tous les vertébrés. II est en effet non fonctionnel dans le génome des oiseaux, des reptiles et des cétartiodactiles (comme le porc). Or, le nœud embryonnaire de ces dernières espèces est couvert par de l'endoderme et non pas par un liquide péri-embryonnaire. La latéralisation chez les oiseaux, les reptiles et les cétartiodactiles serait donc établie par un mouvement circulaire des cellules autour du nœud embryonnaire.

\footnotetext{
${ }^{3}$ Technique permettant de localiser une séquence de nucléotides sur une coupe histologique.

${ }^{4}$ Molécule synthétique antisens d'environ 25 bases qui se fixe aux séquences complémentaires d'ARN par appariement de bases. Le morpholino peut être utilisé pour bloquer la traduction ou modifier l'épissage du préARNm et ainsi diminuer ou invalider l'expression d'un gène.

${ }^{5}$ La pénétrance d'une maladie génétique correspond à la proportion d'individus possédant un génotype donné qui exprime le phénotype correspondant. La pénétrance est incomplète lorsque des individus de génotypes identiques n'expriment pas tous le même phénotype.
} 


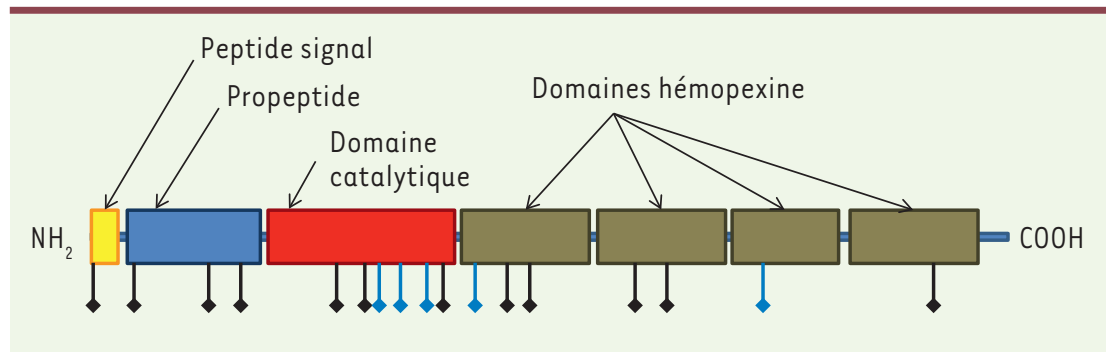

Figure 2. Schéma indiquant les différents domaines de la métalloprotéase MMP21. L'emplacement des mutations est symbolisé en-dessous de la protéine, avec en noir les mutations de l'article de Guimier et al. [5], et en bleu les mutations rapportées dans deux autres articles [6, 7].

\section{Conclusion}

Cette étude a mis en évidence pour la première fois le rôle d'une métalloprotéase dans la latéralisation de la plupart des vertébrés. Le substrat de cette métalloprotéase est à ce jour inconnu. La prévalence élevée $(5,9 \%)$ de mutations de MMP21 dans notre groupe de patients atteints d'hétérotaxie avec ou sans malformation cardiaque place ce gène en première position des gènes responsables d'hétérotaxie devant le gène ZIC3 (zic family member 3 , environ
$3 \%$ des cas). La pénétrance, quoique incomplète, est très élevée (environ $90 \%$ dans notre groupe de patients) et donc bien supérieure à la pénétrance de l'hétérotaxie lorsqu'un gène codant une protéine du cil embryonnaire est impliqué (pénétrance de $50 \%$ ). $\diamond$

A gene coding for a metalloprotease for the first time implied in heterotaxy

\section{LIENS D'INTÉRÊT}

Les auteurs déclarent n'avoir aucun lien d'intérêt concernant les données publiées dans cet article.

\section{RÉFÉRENCES}

1. Bajolle F, Zaffran S. Le débit cardiaque, acteur majeur de la morphogenèse asymétrique des arcs aortiques. Med Sci (Paris) 2008 ; 24 : 354-6.

2. Diguet N, Meilhac SM. Cils et morphogenèse cardiaque. Med Sci (Paris) 2014 ; 30 : 996-1003.

3. Bachmann-Gagescu R. Complexité génétique des ciliopathies et identification de nouveaux gènes. Med Sci (Paris) $2014 ; 30: 1011-23$.

4. Liu H, Jimenez G, દl Malti R, et al. Défauts de latéralisation et hétérotaxie. EMC-Cardiologie 2014 . 9: $1-14$

5. Guimier A, Gabriel GC, Bajolle F, et al. MMP21 is mutated in human heterotaxy and is required fo normal left-right asymmetry in vertebrates. Nat Genet $2015 ; 47: 1260-3$

6. Akawi N, McRae J, Ansari M, et al. Discovery of four recessive developmental disorders using probabilistic genotype and phenotype matching among 4,125 families. Nat Genet 2015 ; 47 : 1363-9.

7. Perles Z, Moon S, Ta-Shma A, et al. A human laterality disorder caused by a homozygous deleterious mutation in MMP21.J Med Genet 2015; 52 : 840-7.

8. Ahokas K, Lohi J, Lohi H, et al. Matrix metalloproteinase-21, the human orthologue for XMMP, is expressed during fetal development and in cancer. Gene $2002 ; 301$ : 31-41.

9. Li Y, Klena NT, Gabriel GC, et al. Global genetic analysis in mice unveils central role for cilia in congenital heart disease. Nature 2015 ; 521 : 520-4.

10. Tremblay JP. CRISPR, un système qui permet de corriger ou de modifier l'expression de gènes esponsables de maladies héréditaires. Med Sci (Paris) $2015 ; 31: 1014-22$
NOUVELLE

\section{Actine et annexine A2}

\section{Partenaires essentiels pour le contrôle de la sécrétion neuroendocrine}

Marion Gabel ${ }^{1}$, Franck Delavoie ${ }^{2}$, Nicolas Vitale ${ }^{1}$, Marie-France Bader ${ }^{1}$, Sylvette Chasserot-Golaz ${ }^{1}$
> Le fonctionnement des systèmes nerveux et neuroendocrine repose sur une communication cellulaire élaborée soutenue par un trafic membranaire intense. Les molécules impliquées dans cette communication sont stockées dans des granules de sécrétion et libérées dans le milieu extracellulaire par fusion des granules avec la membrane plasmique, un processus appelé exocytose régulée [1]. Dans les cellules neuroendocrines, l'arrimage des granules de sécrétion à la membrane plasmique, puis leur fusion, se font au niveau de sites membranaires dont l'architecture est portée par des lipides spécifiques. Ces domaines lipidiques, aussi appelés radeaux lipidiques, correspondent aux sites d'exocytose de la membrane plasmique et peuvent se former de façon transitoire selon l'état d'activation des cellules [2]. Ils recrutent, activent ou séparent les acteurs de l'exocytose comme les protéines du complexe SNARE (soluble $\mathrm{N}$-ethylmaleimide-sensitive factor attachment protein receptor),
${ }^{1}$ Institut des neurosciences cellulaires et intégratives, CNRS - UPR 3212 et université de Strasbourg, 5, rue Blaise Pascal, F-67084, Strasbourg, France.

${ }^{2}$ Laboratoire de biologie moléculaire eucaryote, UMR5099 CNRS-université de Toulouse III Paul Sabatier, 118, route de Narbonne, F-31000, Toulouse, France.

chasserot@inci-cnrs.unistra.fr

nécessaires à l'arrimage et à la fusion des granules à la membrane plasmique $[3,4](\rightarrow)$.

L'annexine $A 2$ a été l'une des premières protéines identifiées au niveau des sites d'exocytose [5]. Elle fait partie $(\rightarrow)$ Voir la Synthèse de T. Galli et al. $m / s n^{\circ} 11$, novembre 2002, page 1113 et la Nouvelle de 0 . El Far et $M$. Seagar, $\mathrm{m} / \mathrm{s} \mathrm{n}^{\circ} 1$, janvier 2011, page 28 de la famille des annexines, protéines capables de s'associer aux membranes biologiques en présence de calcium. Une des particularités de l'annexine A2 